\title{
Measuring Cerebral and Cerebellar Glutathione in Children Using 'H MEGA-PRESS MRS
}

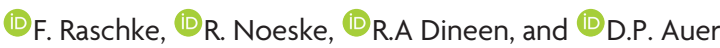

\begin{abstract}
BACKGROUND AND PURPOSE: Glutathione is an important antioxidant in the human brain and therefore of interest in neurodegenerative disorders. The purpose of this study was to investigate the feasibility of measuring glutathione in healthy nonsedated children by using the ${ }^{1} \mathrm{H}$ Mescher-Garwood point-resolved spectroscopy (MEGA-PRESS) sequence at 3T and to compare glutathione levels between the medial parietal gray matter and the cerebellum.
\end{abstract}

MATERIALS AND METHODS: Glutathione was measured using MEGA-PRESS MRS (TR $=1.8$ seconds, TE $=131$ ms) in the parietal gray matter $\left(35 \times 25 \times 20 \mathrm{~mm}^{3}\right)$ of 6 healthy children (10.0 \pm 2.4 years of age; range, $7-14$ years; 3 males) and in the cerebellum of 11 healthy children (12.0 \pm 2.7 years of age; range, 7-16 years; 6 males). A postprocessing pipeline was developed to account for frequency and phase variations in the edited ON and nonedited OFF spectra. Metabolites were quantified with LCModel and reported both as ratios and water-scaled values. Glutathione was quantified in the ON-OFF spectra, whereas total NAA, total Cho, total $\mathrm{Cr}$, mlns, Glx, and taurine were quantified in the OFF spectra.

RESULTS: We found significantly higher glutathione, total Cho, total $\mathrm{Cr}$, mlns, and taurine in the cerebellum $(P<.01)$. Glx and total NAA were significantly higher in the parietal gray matter $(P<.01)$. There was no significant difference in glutathione/total $\mathrm{Cr}(P=.93)$ between parietal gray matter and cerebellum.

CONCLUSIONS: We demonstrated that glutathione measurement in nonsedated children is feasible. We found significantly higher glutathione in the cerebellum compared with the parietal gray matter. Metabolite differences between the parietal gray matter and cerebellum agree with published MRS data in adults.

ABBREVIATIONS: GSH = glutathione; MEGA-PRESS = Mescher-Garwood point-resolved spectroscopy; PGM = parietal gray matter; Tau = taurine; $\mathrm{t}-=$ total

$\mathbf{G}$ lutathione $(\mathrm{GSH})$ is an important antioxidant in the human brain $^{1,2}$ and has been shown to be altered in a number of pathologies. $^{3-7}$ Measuring GSH is of interest in neurodegenera-

Received June 22, 2017; accepted after revision September 13.

From the National Center for Tumor Diseases (F.R.), Partner Site Dresden, Dresden, Germany; German Cancer Research Center (F.R.), Heidelberg, Germany; Medical Faculty and University Hospital Carl Gustav Carus (F.R.), Technische Universität Dresden, Dresden, Germany; and Helmholtz Association/Helmholtz Zentrum Dresden Rossendorf (F.R.), Dresden, Germany; GE Healthcare (R.N.), Applied Science Lab Europe, Berlin, Germany; Sir Peter Mansfield Imaging Centre (R.A.D., D.P.A.), University of Nottingham, Nottingham, UK; Radiological Sciences, (R.A.D., D.P.A.), Division of Clinical Neuroscience, School of Medicine, University of Nottingham, Nottingham, UK; and National Institute for Health Research (R.A.D., D.P.A.), Nottingham Biomedical Research Centre, Nottingham, UK.

F.R. and the cost of the pediatric MRI scans were funded by the A-T Children's Project and Action for A-T.

Please address correspondence to: Rob Dineen, PhD, Radiological Sciences, University of Nottingham, Room B1435, Queen's Medical Centre, Nottingham, NG7 2UH, United Kingdom; e-mail: rob.dineen@nottingham.ac.uk

三 Indicates article with supplemental on-line appendix.

Indicates article with supplemental on-line photos.

http://dx.doi.org/10.3174/ajnr.A5457 tive disorders ${ }^{8}$ and may be a potential mechanistic biomarker for oxidative stress-related diseases and the efficacy of antioxidative treatments. Our specific interest is the quantification of GSH in the brains of children affected by neurodegenerative diseases such as ataxia telangiectasia, requiring a robust method to measure GSH levels in nonsedated children.

GSH can, in principle, be measured in vivo with ${ }^{1} \mathrm{H}-\mathrm{MRS}$. However, due to the low concentration of GSH in the healthy human brain and in particular its overlap with higher concentration metabolites, quantification of GSH at $3 \mathrm{~T}$ with conventional MRS sequences is controversial. ${ }^{9-11}$ Spectral editing allows the removal of overlapping resonances for a direct and robust quantification of GSH. ${ }^{12-14}$ While spectral editing can be used to specifically measure GSH, it is more susceptible to subject motion than conventional single-voxel MRS sequences due to longer scan times and because spectral editing is a subtraction technique that relies on consistent data acquisition.

Few studies have reported in vivo GSH values in healthy children, ${ }^{15,16}$ and, to our knowledge, there is no study that specifically 
measured GSH in children using spectral editing techniques or that reported GSH in the cerebellum. Therefore, the goal of this study was to investigate the feasibility of measuring GSH in healthy nonsedated children with the Mescher-Garwood pointresolved spectroscopy (MEGA-PRESS) ${ }^{13,17}$ sequence and to compare GSH levels obtained in the medial parietal gray matter (PGM) with those measured in the cerebellum. To minimize subtraction errors caused by phase and frequency variations, we adapted a MEGA-PRESS postprocessing technique published by An et $\mathrm{al}^{17}$ and modified it to work with our data.

\section{MATERIALS AND METHODS Subjects}

Healthy children were recruited as part of an ongoing study, approved by the UK National Research Ethics Service East Midlands-Derby Committee (Reference 14/EM/1175). Informed consent was obtained from parents or guardians of participants. MRS data in the PGM were acquired in 6 children $(10.0 \pm 2.4$ years of age; range, 7-14 years; 3 males). MR spectroscopic data in the cerebellum were acquired in 11 children $(12.0 \pm 2.7$ years of age; range, 7-16 years; 6 males). There was no significant difference in age between the 2 groups ( $t$ test, $P=.14$ ). Structural MRI was checked by a neuroradiologist (R.A.D.) to ensure that children were neuroradiologically healthy.

\section{Data Acquisition}

Data were acquired on a 3T MR scanner (Discovery MR750; GE Healthcare, Milwaukee, Wisconsin) equipped with a 32-channel head coil, without sedation. In addition to standard pediatric MRI preparation, younger participants were shown an animation to help prepare them for the MRI, ${ }^{18}$ and participants could watch videos on an MRI-compatible monitor during the scan to improve tolerance. The MRI protocol included a 3D fast-spoiled gradient recalled T1-weighted structural MRI with 1-mm isotropic resolution for MRS planning $(\mathrm{TR}=8.15 \mathrm{~ms}, \mathrm{TE}=3.172 \mathrm{~ms}$, $\mathrm{TI}=900 \mathrm{~ms}, \mathrm{FOV}=256 \times 256 \times 156 \mathrm{~mm})$. Single-voxel MEGAPRESS GSH editing was performed using $\mathrm{TR}=2$ seconds, $\mathrm{TE}=$ $131 \mathrm{~ms}$, and $128 \mathrm{ON}$ and $128 \mathrm{OFF}$ acquisitions. Spectral editing was achieved with sinc-weighted Gaussian pulses with a pulse length of $20 \mathrm{~ms}$ (bandwidth, $64 \mathrm{~Hz}$ ) applied at $7.5 \mathrm{ppm}$ (OFF) and $4.54 \mathrm{ppm}(\mathrm{ON})$ for editing GSH. ${ }^{13,17}$ A nonedited, water-unsuppressed reference scan of 16 averages was acquired at identical acquisition parameters. MRS voxel sizes were $35 \times 25 \times 20 \mathrm{~mm}$ in the PGM and $50 \times 22 \times 22 \mathrm{~mm}$ in the cerebellum. Typical voxel locations are illustrated in Fig 1. Total acquisition time for MEGA-PRESS MRS was 9 minutes 30 seconds.

\section{MRS Processing}

MEGA-PRESS data were processed off-line with Matlab (MathWorks, Natick, Massachusetts). The workflow steps A-H are illustrated in On-line Fig 1. The phase angles between the 32 coil elements were calculated from the average unsuppressed water signal (A). All ON and OFF spectra were subsequently phased using the water phase angles and coil-combined by using the maximum peak height of the unsuppressed water signal as weighting factors (B). The resulting coil-combined $128 \mathrm{ON}$ and $128 \mathrm{OFF}$ spectra were potentially out of phase relative to each other due to subject motion and frequency drift. To correct for this, we phased individual ON and OFF spectra by maximizing the correlation of the NAA peak between the real and absolute part of the spectrum in the range of 1.87 and $2.21 \mathrm{ppm}(\mathrm{C})$. Next, a reference $\mathrm{ON}$ spectrum for phase and frequency correction was created, like that described by An et $\mathrm{al}^{17}$ by pair-wise aligning the $128 \mathrm{ON}$ spectra in the spectral range of 1.3 and $3.3 \mathrm{ppm}$ and iterative pair-wise averaging of the spectra with the smallest root mean square error (D). All $128 \mathrm{ON}$ and 128 OFF spectra were subsequently aligned to this ON reference spectrum by time domain phase and frequency correction using the fminsearch function (https://de.mathworks.com/help/matlab/ref/ fminsearch.html) (E) in Matlab. For the alignment, the correlation coefficient of the real part of the NAA peak (1.87-2.21 ppm) between the $\mathrm{ON}$ reference spectrum and the individual $\mathrm{ON}$ and $\mathrm{OFF}$ spectra was maximized. The 128 aligned ON and OFF spectra were then averaged to $1 \mathrm{ON}$ and $1 \mathrm{OFF}$ spectrum each (F). Individual poor-quality $\mathrm{ON}$ and $\mathrm{OFF}$ spectra were detected by calculating the correlation coefficient of the NAA peak (1.87-2.21 ppm) between the $128 \mathrm{ON} / \mathrm{OFF}$ spectra and the averaged ON/ OFF spectrum, respectively $(\mathrm{G})$. In our data, a correlation coefficient threshold of 0.8 ( 1 indicating perfect correlation) was used to pair-wise exclude individual $\mathrm{ON}$ and $\mathrm{OFF}$ spectra. Finally, the new $\mathrm{ON}$ and $\mathrm{OFF}$ average spectra were recalculated $(\mathrm{H})$ and subtracted to obtain the final edited ON-OFF spectrum.

\section{Metabolite Quantification}

Unedited MEGA-PRESS OFF spectra were analyzed between 4 and $0.2 \mathrm{ppm}$ with LCModel (Version 6.3-1H; http://www.lcmodel. com/lcmodel.shtml $)^{19}$ using a basis set simulated with TARQUIN (http://tarquin.sourceforge.net/). ${ }^{20}$ The OFF spectra were used to quantify total NAA (tNAA $=\mathrm{NAA}+\mathrm{N}$-acetyl aspartylglutamate), total Creatine $(\mathrm{tCr}=\mathrm{Cr}+$ phosphocreatine), total Choline (tCho $=$ glycerophosphorylcholine + phosphorylcholine), mIns, Glx, and Taurine (Tau). The edited ON-OFF MEGA-PRESS spectra were analyzed in the range of 3.6-0.2 ppm with LCModel, using a measured basis set from GSH and NAA solutions. LCModel parameters for the ON-OFF analysis are given in the On-line Appendix.

Metabolite values are reported as ratios to $\mathrm{tCr}$ and as waterscaled values using the unsuppressed OFF water signal. For the latter, corresponding structural T1WI was segmented via SPM software (http://www.fil.ion.ucl.ac.uk/spm/software/spm12) into gray matter, white matter, and CSF and aligned with the MRS volume with in-house software. Water-scaled metabolite values were corrected for CSF contamination by dividing them by $1-\mathrm{CSF} \%$. Because the complex anatomic structure in the cerebellum would not allow an accurate separation of gray and white matter, we did not take their differences in water content into account. $\mathrm{T} 1$ and $\mathrm{T} 2$ correction was not performed; thus, water-scaled and CSF-corrected metabolite values are reported as institutional units.

A 2-sample $t$ test assuming unequal variances was used to determine significant differences in metabolite values and ratios between the PGM and the cerebellum.

\section{RESULTS}

The proposed GSH processing gave robust results in all spectra. On-line Fig 2 shows the edited ON-OFF spectra and correspond- 

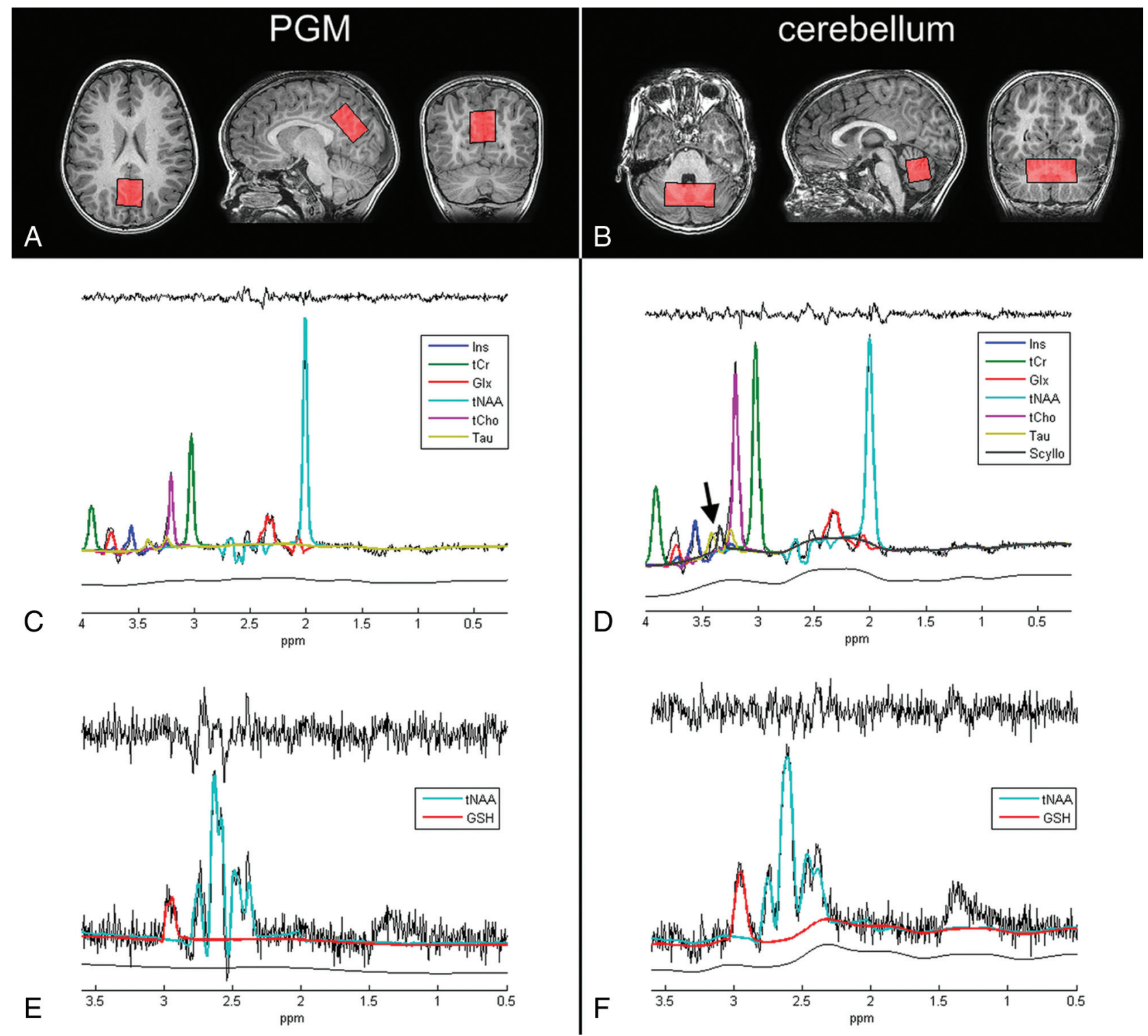

FIG 1. Examples of the MRS voxel placement in the PGM $(A)$ and cerebellum $(B)$ in two 7-year-old children. Corresponding OFF (C and $D)$ and ON-OFF ( $E$ and $F$ ) spectra are shown below. Individual metabolite spectra fitted by LCModel are highlighted in different colors. $D$, The arrow highlights visible resonances that are particularly large in the cerebellum and likely belong to taurine and scyllo-inositol. Residuals of the fits (black, upper row) and estimated LCModel baselines (black, lower row) are shown for each spectrum.

ing GSH fits determined by LCModel for all subjects. An example in On-line Fig 1 illustrates the correction for phase and frequency shifts in a PGM spectrum acquired in an 11-year-old girl.

Figure 1 shows sample spectra from the PGM and cerebellum and corresponding metabolite fits for two 7-year-old children. The OFF spectra from the cerebellum show visible resonances between 3.3 and 3.5 ppm (see the arrow in Fig 1D). LCModel assigned these resonances to taurine (around 3.4 ppm) and scyllo-inositol (around 3.34 ppm), but the fitted scyllo-inositol signals were too small for reliable quantification in both the cerebellum (Cramer-Rao lower bounds, $21 \% \pm 22 \%$ ) and PGM (Cramer-Rao lower bounds, 33\% $\pm 27 \%$ ). We therefore only report the apparent taurine signal for the cerebellum (Cramer-Rao lower bounds, 9\% $\pm 1 \%$ ) and PGM (Cramer-Rao lower bounds, $14 \% \pm 2 \%)$. However, the combined sum of Tau + scyllo-inositol showed the same trends as taurine using either water scaling or ratios (results not shown).
We found significantly higher GSH in the cerebellum compared with the PGM $(P<.01)$. Additionally, we found higher tCho $(P<.01)$, $\operatorname{tCr}(P<.01)$, mIns $(P<.01)$, and Tau $(P<.01)$ in the cerebellum, whereas NAA $(P<.01)$ and Glx $(P<.01)$ were significantly higher in the PGM. Boxplots are shown in Fig 2.

Figure 3 shows the results of the metabolite ratios between the $\mathrm{PGM}$ and cerebellum. There was no significant difference in GSH/ $\mathrm{tCr}(P=.93)$. However, tNAA/tCr $(P<.01)$, tNAA/tCho $(P<$ $.01)$, mIns/tCr $(P=.01)$, and $\mathrm{Glx} / \mathrm{tCr}(P<.01)$ were significantly higher in the PGM. Both $\mathrm{tCho} / \mathrm{tCr}(P<.01)$ and Tau/tCr $(P=$ .02) were lower in the PGM.

\section{DISCUSSION}

We demonstrate reliable detection of glutathione levels in the parietal gray matter and cerebellum of nonsedated children using a dedicated proton MRS protocol for acquisition and postprocessing. Postpro- 

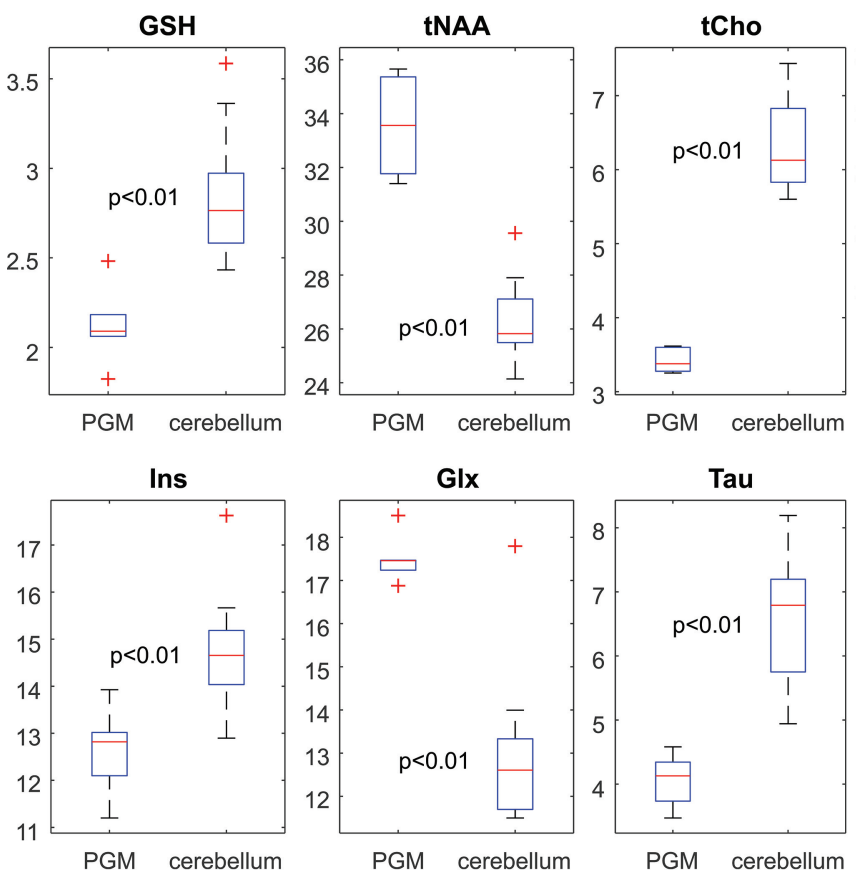

FIG 2. Boxplots showing the water-scaled metabolite values in institutional units in the PGM and cerebellum.
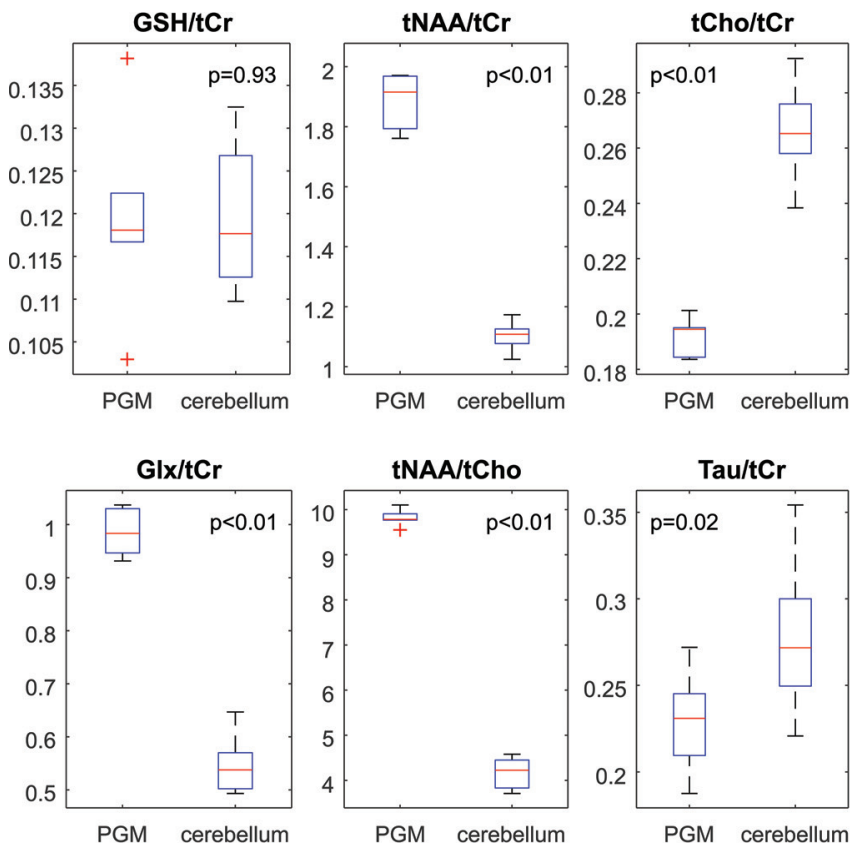

FIG 3. Boxplots showing the metabolite ratios in the PGM and cerebellum.
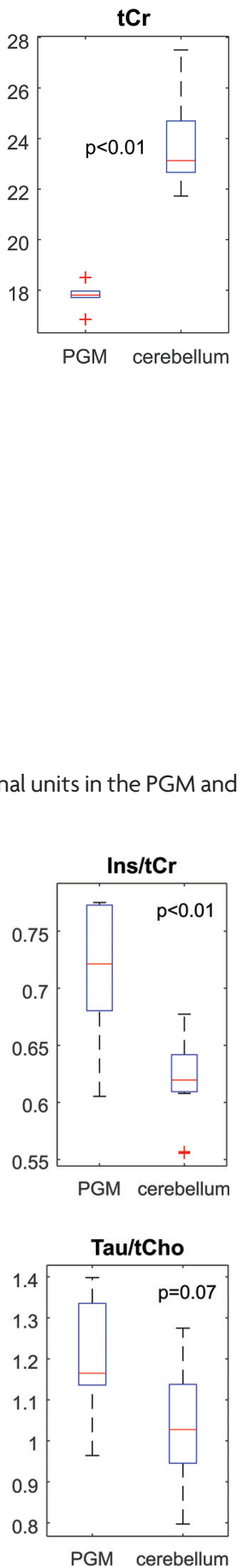

omitting zero and first-order baseline adjustments. This was performed to reduce the number of fitted parameters for a more robust parameter determination in our lower SNR spectra caused by a smaller voxel size.

To the best of our knowledge, this is the first study to compare GSH levels in the cerebrum with those found in the cerebellum in children. Few studies compared GSH levels between the cerebellum and the cerebrum in adults. In agreement with our findings, Emir et $\mathrm{al}^{21}$ found higher GSH and $\mathrm{tCr}$ in the vermis compared with the occipital cortex and posterior cingulate in healthy adults using a short-echo STEAM sequence at 7T, albeit without T1, T2, and CSF correction. An extensive postmortem study in humans by Tong et $\mathrm{al}^{22}$ showed no significant difference in GSH between the occipital and cerebellar cortices in the 1- to 18-year age group. A histologic study in mice by Kang et $\mathrm{al}^{23}$ revealed slightly lower GSH in the cerebellum compared with the cortex.

Previous studies reported higher Cho and $\mathrm{Cr}$ levels in the cerebellum compared with the cerebrum in healthy adults, ${ }^{24,25}$ in agreement with the results in our cohort of children, whereas an MR spectroscopic imaging study by Lecocq et $\mathrm{al}^{26}$ found only reduced NAA in the cerebellum. Looking at metabolite ratios, several studies found lower $\mathrm{tNAA} / \mathrm{tCr}$ in the cerebellum compared with the cerebrum in children ${ }^{27}$ and adults, ${ }^{28-30}$ in agreement with our findings. Additionally, Goryawala et $\mathrm{al}^{31}$ showed lower Glx/tCr in the cerebellum in adults, in agreement with our results in children. Another noteworthy finding in this study was higher apparent $\mathrm{Tau} / \mathrm{tCr}$ in the cerebellum compared with the PGM. Taurine is particularly high in infants and children in the cerebellar cortex. ${ }^{32,33}$ Additionally, elevated taurine is characteristic of medulloblastomas, ${ }^{34}$ mainly originating in the cerebellum of children.

A limitation of this study is the relatively small sample size and the use of different subjects to scan the PGM and the cerebellum. Scanning the PGM and cerebellum in each subject would have allowed a pair-wise statistical analysis and likely reduced variability. We were, however, limited by time constraints in the MRI protocol due to the long acquisition time of GSH MEGA-PRESS MRS. Additionally, the MRS voxel in the cerebellum was relatively large, to ensure high-enough SNR for GSH quantification, thus having a relatively heterogeneous tissue composition, including the cerebellar gray matter (vermis and cortex) and the underlying cerebellar white matter. 


\section{CONCLUSIONS}

We demonstrated that GSH measurement in nonsedated children is feasible. We found higher GSH in the cerebellum compared with the PGM. Differences in the other metabolites agree with published MRS data in adults; this finding suggests no major metabolic maturation effect from 7 years of age and older in our dataset.

Disclosures: Felix Raschke—RELATED: Grant: A-T Children's Project and Action for A-T, Comments: This work was supported by a research grant awarded by the 2 named charities*; UNRELATED: Support for Travel to Meetings for the Study or Other Purposes: Guarantors of Brain, Comments: travel grant to support travel to the International Society for Magnetic Resonance in Medicine meeting in 2015. Ralph Noeske-UNRELATED: Employment: GE Healthcare. Robert A. Dineen-RELATED: Grant: A-T Children's Project and Action for A-T.* Dorothee P. Auer-RELATED: Grant: A-T Children's Project and Action for A-T*; UNRELATED: Grants/Grants Pending: UK charities, UK research councils, National Institute for Health Research, UK funding. * *Money paid to the institution.

\section{REFERENCES}

1. Dringen R. Metabolism and functions of glutathione in brain. Prog Neurobiol 2000;62:649-71 CrossRef Medline

2. Mazzetti AP, Fiorile MC, Primavera A, et al. Glutathione transferases and neurodegenerative diseases. Neurochem Int 2015;82: 10-18 CrossRef Medline

3. An L, Dani KA, Shen J, et al; Natural History of Stroke Investigators. Pilot results of in vivo brain glutathione measurements in stroke patients. J Cereb Blood Flow Metab 2012;32:2118-21 CrossRef Medline

4. Choi IY, Lee SP, Denney DR, et al. Lower levels of glutathione in the brains of secondary progressive multiple sclerosis patients measured by $1 \mathrm{H}$ magnetic resonance chemical shift imaging at $3 \mathrm{~T}$. Mult Scler 2011;17:289-96 CrossRef Medline

5. Do KQ, Trabesinger AH, Kirsten-Krüger M, et al. Schizophrenia: glutathione deficit in cerebrospinal fluid and prefrontal cortex in vivo. Eur J Neurosci 2000;12:3721-28 CrossRef Medline

6. Matsuzawa D, Obata T, Shirayama Y, et al. Negative correlation between brain glutathione level and negative symptoms in schizophrenia: a 3T 1H-MRS study. PLoS One 2008;3:e1944 CrossRef Medline

7. Srinivasan R, Ratiney KH, Hammond-Rosenbluth KE, et al. MR spectroscopic imaging of glutathione in the white and gray matter at $7 \mathrm{~T}$ with an application to multiple sclerosis. Magn Reson Imaging 2010; 28:163-70 CrossRef Medline

8. Aoyama K, Nakaki T. Impaired glutathione synthesis in neurodegeneration. Int J Mol Sci 2013;14:21021-44 CrossRef Medline

9. Sanaei Nezhad F, Anton A, Parkes LM, et al. Quantification of glutathione in the human brain by MR spectroscopy at 3 Tesla: comparison of PRESS and MEGA-PRESS. Magn Reson Med 2017;78: 1257-66 CrossRef Medline

10. Oz G, Tkáč I. Short-echo, single-shot, full-intensity proton magnetic resonance spectroscopy for neurochemical profiling at $4 \mathrm{~T}$ : validation in the cerebellum and brainstem. Magn Reson Med 2011; 65:901-10 CrossRef Medline

11. Mekle R, Mlynárik V, Gambarota G, et al. MR spectroscopy of the human brain with enhanced signal intensity at ultrashort echo times on a clinical platform at 3T and 7T. Magn Reson Med 2009;61: 1279-85 CrossRef Medline

12. Mescher M, Merkle H, Kirsch J, et al. Simultaneous in vivo spectral editing and water suppression. NMR Biomed 1998;11:266-72 Medline

13. Terpstra M, Henry PG, Gruetter R. Measurement of reduced glutathione (GSH) in human brain using LCModel analysis of difference-edited spectra. Magn Reson Med 2003;50:19-23 CrossRef Medline

14. Trabesinger AH, Weber OH, Duc CO, et al. Detection of glutathione in the human brain in vivo by means of double quantum coherence filtering. Magn Reson Med 1999;42:283-89 Medline

15. Degnan AJ, Ceschin R, Lee V, et al. Early metabolic development of posteromedial cortex and thalamus in humans analyzed via in vivo quantitative magnetic resonance spectroscopy. J Comp Neurol 2014; 522:3717-32 CrossRef Medline

16. Kreis R, Hofmann L, Kuhlmann B, et al. Brain metabolite composition during early human brain development as measured by quantitative in vivo $1 \mathrm{H}$ magnetic resonance spectroscopy. Magn Reson Med 2002;48:949-58 CrossRef Medline

17. An L, Zhang Y, Thomasson DM, et al. Measurement of glutathione in normal volunteers and stroke patients at $3 \mathrm{~T}$ using J-difference spectroscopy with minimized subtraction errors. J Magn Reson Imaging 2009;30:263-70 CrossRef Medline

18. Szeszak S, Man R, Love A, et al. Animated educational video to prepare children for MRI without sedation: evaluation of the appeal and value. Pediatr Radiol 2016;46:1744-50 CrossRef Medline

19. Provencher SW. Estimation of metabolite concentrations from localized in vivo proton NMR spectra. Magn Reson Med 1993;30: 672-79 CrossRef Medline

20. Wilson M, Reynolds G, Kauppinen RA, et al. A constrained leastsquares approach to the automated quantitation of in vivo ${ }^{1} \mathrm{H}$ magnetic resonance spectroscopy data. Magn Reson Med 2011;65:1-12 CrossRef Medline

21. Emir UE, Auerbach EJ, Van De Moortele PF, et al. Regional neurochemical profiles in the human brain measured by ${ }^{1} \mathrm{H}$ MRS at $7 \mathrm{~T}$ using local B1 shimming. NMR Biomed 2012;25:152-60 CrossRef Medline

22. Tong J, Fitzmaurice PS, Moszczynska A, et al. Do glutathione levels decline in aging human brain? Free Radic Biol Med 2016;93:110-17 CrossRef Medline

23. Kang $Y$, Viswanath $\mathrm{V}$, Jha $\mathrm{N}$, et al. Brain gamma-glutamyl cysteine synthetase (GCS) mRNA expression patterns correlate with regional-specific enzyme activities and glutathione levels. J Neurosci Res 1999;58: 436-41 Medline

24. Pouwels PJ, Frahm J. Regional metabolite concentrations in human brain as determined by quantitative localized proton MRS. Magn Reson Med 1998;39:53-60 CrossRef Medline

25. Jacobs MA, Horská A, van Zijl PC, et al. Quantitative proton MR spectroscopic imaging of normal human cerebellum and brain stem. Magn Reson Med 2001;46:699-705 Medline

26. Lecocq A, Le Fur Y, Maudsley AA, et al. Whole-brain quantitative mapping of metabolites using short echo three-dimensional proton MRSI. J Magn Reson Imaging 2015;42:280-89 CrossRef Medline

27. Cichocka M, Kozub J, Karcz P, et al. Regional differences in the concentrations of metabolites in the brain of healthy children: a proton magnetic resonance spectroscopy ((1)HMRS) study. Polish J Radiol 2016;81:473-77 CrossRef Medline

28. Granata F, Pandolfo G, Vinci S, et al. Proton magnetic resonance spectroscopy (H-MRS) in chronic schizophrenia: a single-voxel study in three regions involved in a pathogenetic theory. Neuroradiol J 2013;26:277-83 CrossRef Medline

29. Safriel Y, Pol-Rodriguez M, Novotny EJ, et al. Reference values for long echo time MR spectroscopy in healthy adults. AJNR Am J Neuroradiol 2005;26:1439-45 Medline

30. Sabati M, Sheriff S, Gu M, et al. Multivendor implementation and comparison of volumetric whole-brain echo-planar MR spectroscopic imaging. Magn Reson Med 2015;74:1209-20 CrossRef Medline

31. Goryawala MZ, Sheriff S, Maudsley A. Regional distributions of brain glutamate and glutamine in normal subjects. NMR Biomed 2016;29:1108-16 CrossRef Medline

32. Pouwels PJ, Brockmann K, Kruse B, et al. Regional age dependence of human brain metabolites from infancy to adulthood as detected by quantitative localized proton MRS. Pediatr Res 1999;46:474-85 CrossRef Medline

33. Blüml S, Wisnowski JL, Nelson MD Jr, et al. Metabolic maturation of the human brain from birth through adolescence: insights from in vivo magnetic resonance spectroscopy. Cereb Cortex 2013;23: 2944-55 CrossRef Medline

34. Davies NP, Wilson M, Harris LM, et al. Identification and characterisation of childhood cerebellar tumours by in vivo proton MRS. NMR Biomed 2008;21:908-18 CrossRef Medline 